\title{
Adaptive Variable Structure Controller Using Neural Networks
}

\author{
Hussain. N. Al-Duwaish*, and Zakariya. M. Al-Hamouz \\ Department of Electrical Engineering \\ KFUPM, Dhahran 31261, Saudi Arabia. \\ *e-mail: hduwaish@kfupm.edu.sa
}

\begin{abstract}
PSS) of a single machine power system model has been designed using a neural network based variabie structure controller ( VSC). The need for adaptive VSC comes from the fact that the power system model operates over a wide range of operating points, some of which are unstable, and hence no single VSC gains are sufficient for the entire operating range. Neural networks are used for on-line prediction of the suitable VSC gains when the operating point changes. Simulation results are included to demonstrate the performance of the proposed control scheme.
\end{abstract}

\section{INTRODUCTION}

In recent years, there is an ongoing interest on the application of the variable structure controllers (VSC) to different engineering problems including power systems[112], aerospace [13-15], robotics [16-17], and many others. The VSC is essentially a switching feedback control where the gains in each feedback path switch between two values according to some rule. The switching feedback law drives the controlled system's state trajectory onto specified surface called the sliding surface which represent the desired dynamic behavior of the controlled system. The advantage of switching between different feedback structures is to combine the useful properties of each structure and to introduce new properties that are not present in any of the structures used. The design of VSC involves finding the switching vectors representing the sliding surface and the feedback gains.

The switching vectors are very important in improving the system dynamic performance. Selection of switching vectors can be done by pole placement or linear optimal control theory. Feedback gains selection represents the second step in the design of VSC. The objective of this step is to find the appropriate feedback gains that will drive the system's state trajectory to the switching surface defined by the switching vectors. Recently, genetic algorithms have been used for VSC feedback gains selection [18-20].

The feedback gain selection of the VSC is normally based on one operating point and its performance away from the design operating point is, of necessity, a compromise. The limitations imposed on the effectiveness of the VSC by different operating conditions can be overcome by using adaptive control techniques. In this paper, an adaptive output feedback controller using neural networks is proposed to enhance the performance of VSC under different operating points. A neural network is trained to update the feedback gain when the operating point changes.

\section{THEORY OF VSC}

The fundamental theory of variable structure systems may be found in [21]. Different control goals such as stabilization, tracking, regulation can be achieved using VSC by the proper design of the sliding surface. The discussion here will be limited to the regulation problem where the objective is to keep specified states as close to zero as possible. A block diagram of the VSC for the regulation problem is shown in Fig. 1. The control law is a linear state feedback whose coefficients are piecewise constant functions. Consider the linear time-invariant controllable system given by

$$
\dot{X}(t)=A X(t)+B U(t)
$$

Where

$X(t)$ n-dimensional state vector

$U(\mathrm{t}) m$-dimensional control force vector

A $n \times n$ system matrix

$B \quad n \times m$ input matrix

The VSC control laws for the system of (1) are given by

$u_{i}=-\psi_{i}^{T} X=-\sum_{j=1}^{n} \psi_{i j} x_{j} ; i=1,2, \ldots ., m$

Where the feedback gains are given as

$\psi_{i j}=\left\{\begin{array}{l}\left.\alpha_{i j}, \text { if } x_{i} \sigma_{j}\right\rangle 0 ; i=1, \ldots, m \\ -\alpha_{i j}, \text { if } x_{j} \sigma_{i}\langle 0 ; j=1, \ldots, n\end{array}\right.$

and

$$
\sigma_{i}(X)=C_{i}^{T} X=0, \quad i=1, \ldots ., m
$$

Where $C_{i}$ 's are the switching vectors which are selected by pole placement or linear optimal control theory.

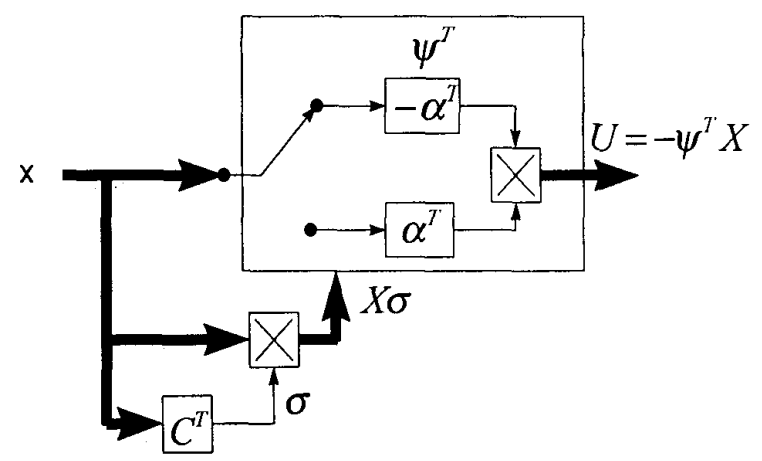

Fig. 1. Block diagram of variable structure controller 
The design procedure for selecting the constant switching vectors $C_{i}$ using pole placement is described below .

Step1: Define the coordinate transformation

$$
\boldsymbol{Y}=\boldsymbol{M} \boldsymbol{X}
$$

such that

$$
M B=\left[\begin{array}{l}
0 \\
\cdots \\
B_{2}
\end{array}\right]
$$

where $M$ is a nonsingular $n \times n$ matrix and $B_{2}$ is a nonsingular $m \times m$ matrix.

From (4) and (5)

$$
\dot{Y}=M \dot{X}=M A M^{-1} Y+M B U
$$

Equation (6) can be written in the form

$$
\left[\begin{array}{l}
\dot{Y}_{1} \\
\dot{Y}_{2}
\end{array}\right]=\left[\begin{array}{ll}
A_{11} & A_{12} \\
A_{21} & A_{22}
\end{array}\right]\left[\begin{array}{l}
Y_{1} \\
Y_{2}
\end{array}\right]+\left[\begin{array}{l}
0 \\
B_{2}
\end{array}\right] U
$$

where $A_{11}, A_{12}, A_{21}, A_{22}$ are respectively $(n-m) \times(n-m)$, $(n-m) \times m, m \times(n-m)$ and $(m \times m)$ submatrices. The first equation of (7) together with (3) specifies the motion of the system in the sliding modem that is

$$
\begin{aligned}
& \dot{Y_{1}}=A_{11} Y_{1}+A_{12} Y_{2} \\
& \sum(Y)=C_{11} Y_{1}+C_{12} Y_{2}
\end{aligned}
$$

where $C_{11}$ and $C_{12}$ are $m \times(n-m)$ and $(m \times m)$ matrices, respectively satisfying the relation

$$
\left[C_{11} C_{12}\right]=C^{T} M^{-1}
$$

Equations (8) and (9) uniquely determine the dynamics in the sliding mode over the intersection of the switching hyperplanes

$$
\sigma_{i}(X)=C_{i}^{T} X=0, \quad i=1, \ldots, m
$$

The subsystem described by (8) may be regarded as an open loop control system with state vector $Y_{1}$ and control vector $Y_{2}$ being determined by ( 9 ), that is

$$
Y_{2}=-C_{12}^{-1} C_{11} Y_{1}
$$

Consequently, the problem of designing a system with desirable properties in the sliding mode can be regarded as a linear feedback design problem. Therefore, it can be assumed, without loss of generality, that $C_{12}=$ identity matrix of proper dimension.

Step 2: Equations (8) and (11) can be combined to obtain

$$
\dot{Y}_{1}=\left[A_{11}-A_{12} C_{11}\right] Y_{1}
$$

Utkin and Yang [22] have shown that if the pair (A, B) is controllable, then the pair $\left(A_{11}, A_{12}\right)$ is also controllable. If the pair $\left(A_{11}, A_{12}\right)$ is controllable, then the eigenvalues of the matrix $\left[A_{11}-A_{12} C_{11}\right]$ in the sliding mode can be placed arbitrarily by suitable choice of $C_{11}$. The feedback gains $\alpha_{i j}$ are usually determined by simulating the control system and trying different values until satisfactory performance is obtained.

\section{MULTILAYER NEURAL NETWORKS WITH BACK PROPAGATION LEARNING}

In this section, the fundamentals and the architecture of multilayer feedforward neural networks are described.

A multilayer neural network is a layered network consisting of an input layer, an output layer, and one or more hidden layers. Each layer consists of a set of neurons which are fully connected to the neurons in the next layer. The connections have multiplying weights associated with them. The number of neurons and hidden layers is problem-dependent. However, it has been proved that one hidden layer can perform any nonlinear and no more than two hidden layers are needed [23]. A multilayer feedforward neural network with one hidden layer is shown in Fig.2. The connection weights between the neurons and thresholds are determined using the generalized delta rule [24]. The process of determining the weights is called training or learning process. The training process requires a set of input and output patterns. The patterns are fed into the neural networks. The neurons in the input layer receive input signals, the activation signals propagate forward, through the hidden layer(s), to the output layer. The output layer then gives the desired output. The network learns by comparing its output of each input pattern with the actual output of that pattern. The error (the difference between the actual outputs and the predicted outputs of the network) is calculated and propagated backwards from the output to the hidden layer to the input. This is done by minimizing the error function:

$E=\sum_{p} E_{p}=\frac{1}{2} \sum_{p} \sum_{k}\left(t_{k}^{(p)}-y_{k}^{(p)}\right)^{2}$

where $t_{k}$ is the actual output and $y_{k}$ is the predicted output of the neural network.

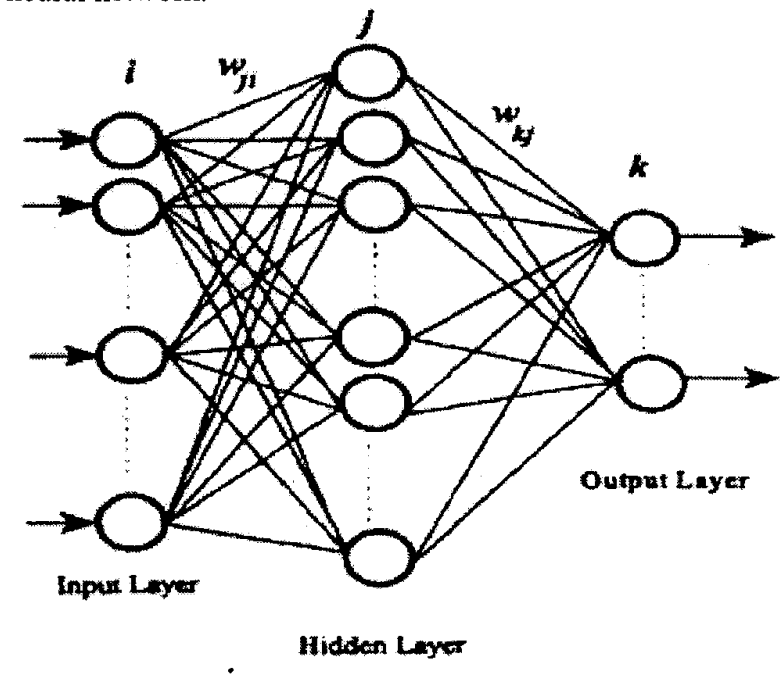

Figure 2: A multilayer feedforward neural network 
Normally, the activation functions are taken as tangential sigmoid and linear for the hidden and output layers, respectively. Since the input neurons have an activation function of unity, then, for each neuron $j$ in the hidden layer, the input is given by

$n e t_{j}^{(p)}=\sum_{i} w_{j i} y_{i}^{(p)}$

where $i$ is a neuron in the input layer, $y_{i}$ is the output of neuron $i, w_{j i}$ is the connection weight from neuron $i$ to neuron $j$, and $p$ represents a training pattern. The output of neuron $j$ is given by

$y_{j}^{(p)}=f_{j}\left(n e t_{j}^{(p)}, \theta_{j}\right)=\frac{\left[1-\exp \left(-\left(n e t_{j}^{(p)}+\theta_{j}\right)\right)\right]}{\left[1+\exp \left(-\left(n e t_{j}^{(p)}+\theta_{j}\right)\right)\right]}$

where $\theta_{j}$ is a bias or threshold at neuron $j$. The output of a neuron $k$ in the output layer is chosen to be a linear sum of all inputs coming from the neurons of the hidden layer, that is

$y_{k}^{(p)}=\sum_{j} w_{k j} y_{j}^{(p)}$

After calculating the actual output using (16), the weights are adapted to reduce the output error. The connection weights between the hidden neuron $j$ and an output neuron $k$ are updated using the following equation:

$\Delta w_{k j}^{(p)}=\eta \delta_{k}^{(p)} y_{k}^{(p)}+\alpha \Delta w_{k j}^{(p-1)}$

where

$\delta_{k}^{(p)}=\left(t_{k}^{(p)}-y_{k}^{(p)}\right) f_{k}\left(n e t_{k}\right)=\left(t_{k}^{(p)}-y_{k}^{(p)}\right) \sum_{j} y_{j}$

The constant $\eta$ is the learning rate and $\alpha$ is the momentum.

The connection weight between an input neuron $i$ and a hidden neuron $j$ can be updated using (19):

$\Delta w_{j i}^{(p)}=\eta \delta_{j}^{(p)} y_{i}^{(p)}+\alpha \Delta w_{j i}^{(p-1)}$

where

$\delta_{j}^{(p)}=f_{j}\left(n e t_{j}^{(\rho)}\right) \sum_{k} \delta_{k}^{(p)} w_{k j}$

\section{Adaptive VSC and Simulation Results}

The proposed method is applied the design of a power system stabilizer (PSS) of a single machine power system model. Fig. 3 shows the block diagram of the linearized power system model for low-frequency oscillation studies. The dynamic model in state-variable form can be obtained from the transfer function model and is given as [25]

$$
\dot{X}(t)=A X(t)+B u(t)+F d(t)
$$

where

$$
\begin{aligned}
& X(t)=\left[\begin{array}{llll}
\Delta \omega(t) & \Delta \delta(t) & \Delta e_{q}^{\prime}(t) & \Delta e_{f d}(t)
\end{array}\right]^{T}, \\
& u(t)=u_{(\text {from } V S C)}, d(t)=\Delta T_{m}(t)
\end{aligned}
$$

$$
\begin{aligned}
& A=\left[\begin{array}{cccc}
-\frac{D}{M} & -\frac{K_{1}}{M} & -\frac{K_{2}}{M} & 0 \\
\omega_{0} & 0 & 0 & 0 \\
0 & -\frac{K_{4}}{T_{d 0}^{\prime}} & -\frac{1}{T_{d 0}^{\prime} K_{3}} & \frac{1}{T_{d 0}^{\prime}} \\
0 & -\frac{K_{4} K_{5}}{T_{A}} & -\frac{K_{4} K_{6}}{T_{A}} & -\frac{1}{T_{A}}
\end{array}\right] \\
& B=\left[\begin{array}{llll}
0 & 0 & 0 & \frac{K_{A}}{T_{A}}
\end{array}\right]^{T} \\
& F=\left[\begin{array}{llll}
\frac{1}{M} & 0 & 0 & 0
\end{array}\right]^{T}
\end{aligned}
$$

The system model is a function of the operating point defined by active and reactive powers $(P, Q)$. The need for adaptive VSC comes from the fact that the model discussed operates over a wide range of operating points, some of which are unstable. Thus, no single VSC with fixed feedback gains is sufficient for the entire operation. The neural network is used to model the nonlinear relationships between operating points and VSC feedback gains. When the operating point changes the neural network will produce new feedback gains suitable for the new operating point.

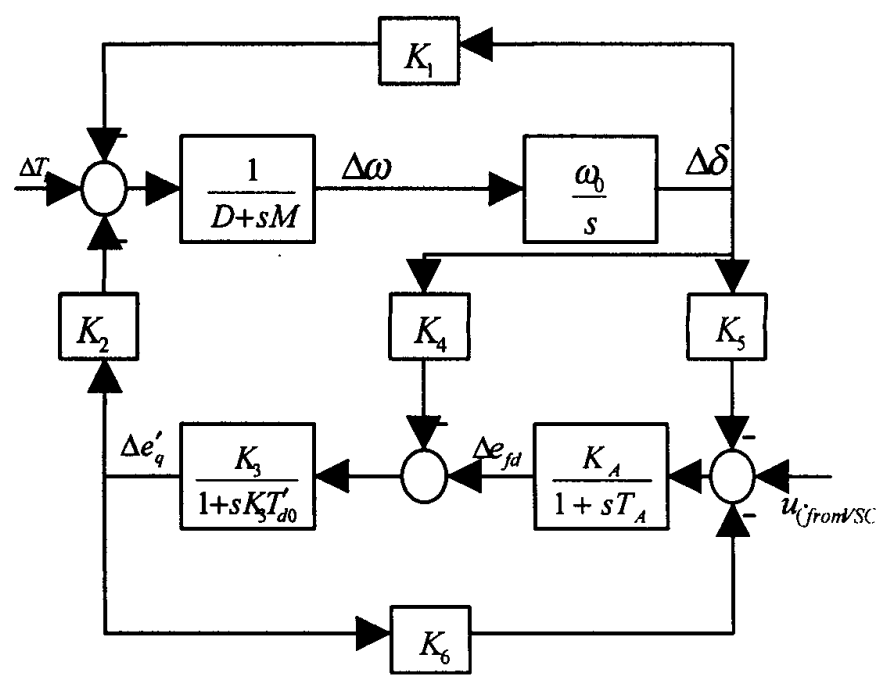

Fig. 3. Block diagram of a single machine power system model

In the adaptive VSC, a neural network with inputs $(P, Q)$ is used to generate the VSC gains $\left(\alpha_{1}\right.$ and $\left.\alpha_{2}\right)$. When the operating point changes, the neural network will produce new VSC gains suitable for the new operating point. To train the neural network, 210 operating points generated by changing $P$ from 0.1 to 1.0 and $Q$ from -1 to 1 in steps of 0.1 , which represent the practical operating range of the studied system, are used. For each operating point, VSC gains $\left(\alpha_{1}\right.$ and $\alpha_{2}$ ) are calculated following the procedure described [18]. The neural network used has two inputs $(P, Q)$, two outputs $\left(\alpha_{1}\right.$ and $\left.\alpha_{2}\right)$, and 30 neurons in the hidden layer. The 
results of the training are shown in figure 4 and figure 5 which indicate good agreement between the actual feedback gains and outputs of the neural network.

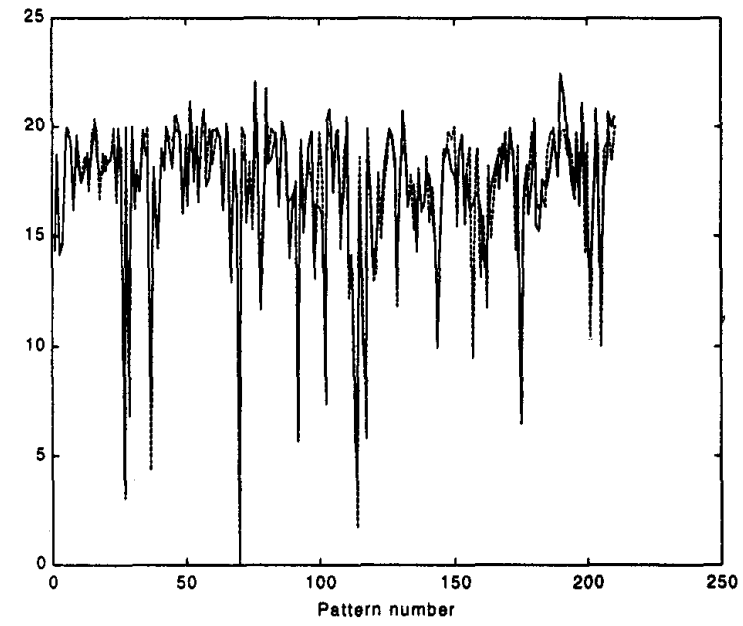

Fig. 4. Actual and predicted values of $\alpha_{1}$

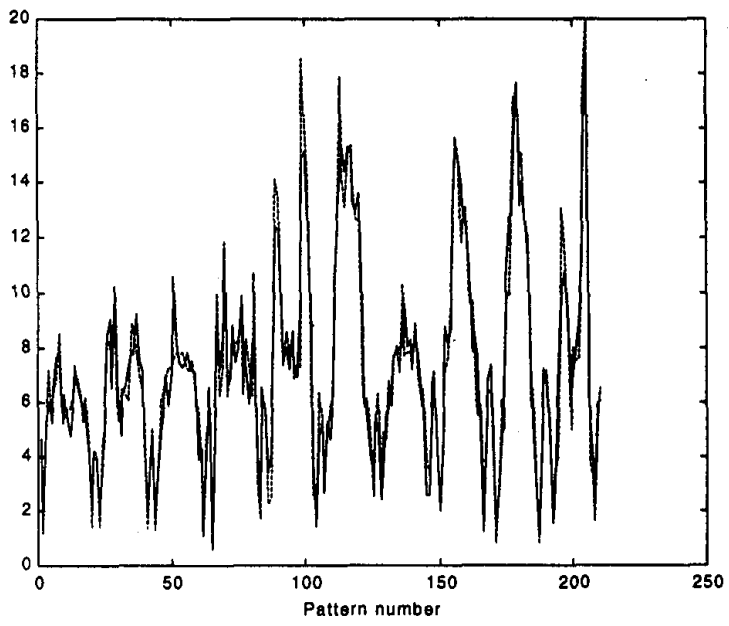

Fig. 5. Actual and predicted values of $\alpha_{2}$

The control objective in the PSS problem is to keep the change in frequency ( $\Delta \omega)$ as close to zero as possible when the operating point changes by manipulating the input $(u)$.

For the operating point of $(P=0.1, Q=1.0)$, a fixed variable structure controller for the above system has been designed. To reduce the complexity of the VSC, the two states $\Delta \omega$ and $\Delta \delta$ are used for feedback. The switching vector is given to be [26]

$$
C=\left[\begin{array}{llll}
-30000 & -97.2134 & 107.0026 & 1
\end{array}\right]^{T}
$$

and the feedback gains obtained using genetic algorithms are

$$
\alpha_{1}=18.5109 \quad, \alpha_{2}=4.2116
$$

Fig. 6 shows the simulation results of the change in frequency $(\Delta \omega)$ when the operating point of the systems changes from $(P=0.1, Q=1.0)$ to $(P=0.3, Q=-0.9)$ at time 10 seconds. The figure demonstrates the effectiveness of the adaptive VSC in damping the frequency oscillations. On the other hand, Fig. 7 shows the change in the torque angle when using the fixed and adaptive VSC. It is quite clear that the adaptive VSC drives the torque angle to its steady state value much faster than the fixed VSC. Figure 8 shows the control efforts of the fixed and adaptive VSC gains. The figure clearly demonstrates the lower control effort needed for the case of adaptive VSC gains.

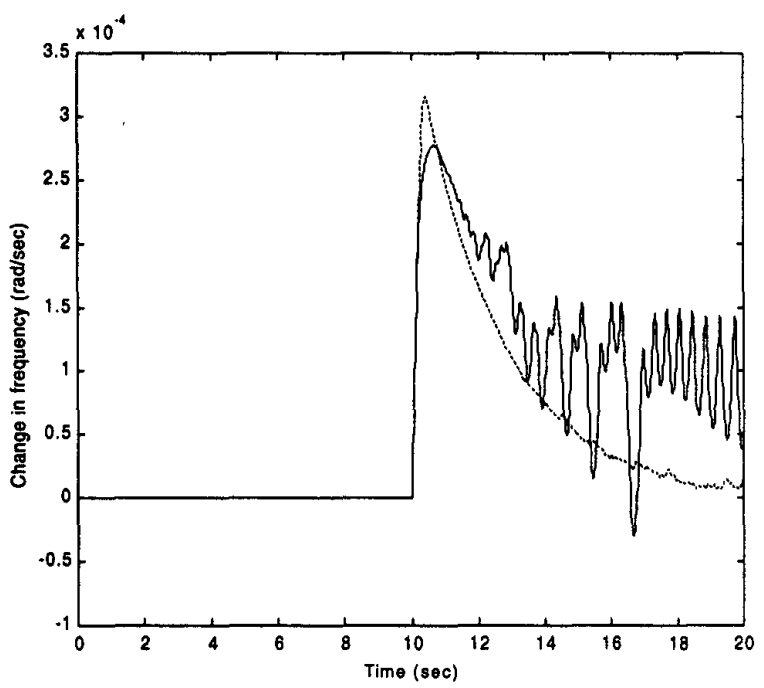

Fig. 6. Change in frequency ( $\Delta \omega)$ for fixed and adaptive VSC gains: (-) for fixed, (...) for adaptive

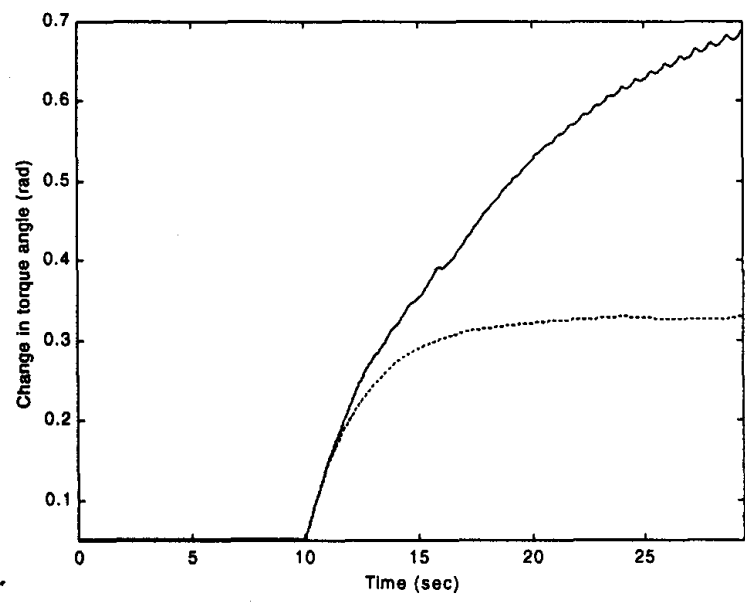

Fig. 7. Change in torque angle ( $\Delta \delta$ )for fixed and adaptive VSC gains: (-) for fixed, (..) for adaptive 


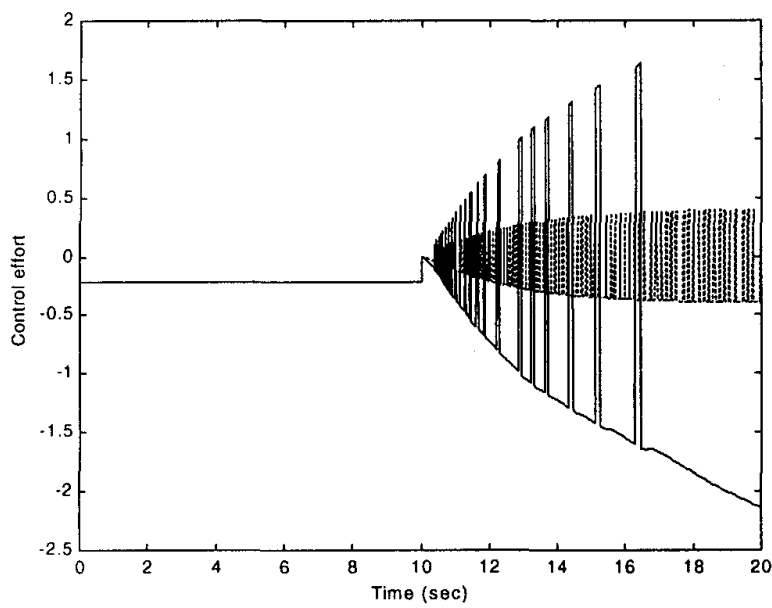

Fig. 8. Control efforts for fixed and adaptive VSC gains: (-) for fixed, (...) for adaptive

\section{CONCLUSION}

In this paper, an adaptive neural network based VSC has been developed for a PSS of a single machine power system. The neural network is used to predict the suitable VSC gains for any operating point. The use of adaptive output feedback is motivated by the fact that the single machine power system operates over a wide range of operating conditions and hence no single VSC gains are sufficient for the entire operation. Simulation results indicate that fixed VSC can perform satisfactorily for a wide range of operating points, but the controller performance can be improved greatly by the use of adaptive VSC.

\section{AKNOWLEDGEMENT}

The authors would like to acknowledge the support of King Fahd University of Petroleum and Minerals.

\section{REFERENCES}

[1] Z. Al-Hamouz and Y. Abdel-Magid, "Variable structure load frequency controllers for multiarea power systems", Int. J. Elect. Power and Energy Syst., Vol. 15, No. 5, pp. 293-300, 1993.

[2] S. Lee, and J. Park, "Design of power system stabilizer using observer/sliding mode, observer/sliding mode-model following and $H_{\infty}$ /sliding mode controllers for small-signal stability study", Electrical Power \&Energy Systems, Vol. 20, No. 8, pp. 543-553, 1998.

[3] A.Y. Sivarmakrishnan, M.A Hariharan and M.C. Srisailam, "Design of variable structure load frequency controller using pole assignment technique", Int. J. Control, Vol. 40, No. 3, pp. 487-498, 1984.

[4] K. Bhattacharya, M. Kothari, and J. Nanda, "Design of discrete mode variable structure power system stabilizers", Int. J. Elect. Power and Energy Syst., Vol. 17, No. 6, pp. 399-406, 1995.

[5] A.S. Murty, S. Parameswaran and K. Ramar, "Design of variable structure stabilizer using pole assignment technique", Electric Machines and Power Systems, Vol.26, No. 3, pp. 185-206, 1998.

[6] Y. Abdel-Magid, Z. Al-Hamouz and J. Bakhashwain, "A variablestructure stabilizer for wind turbine generators", Electric Power Systems Research, Vol. 33, pp. 41-48, 1995.
[7] P. Balasubramanyam, A. Murthy and S. Parameswaran, "Design of variable structure controller for static VAR compensator", Electric Machines and Power Systems, Vol. 26, No. 4, pp. 431-450,1998

[8] V. Samarasinghe and N. Pahalawatha, "Damping of multimodal oscillations in power systems using variable structure control technique", IEE Proc., Generation, Transmission \& Distribution, Vol. 144, No. 3, pp. 323-331, 1997.

[9] T-L Chern, J. Chang, G-K. Chang, "DSP-based integral variable structure model following control for brushless DC motor drivers", IEEE Trans. Power Elect., Vol. 12, No.1, pp. 53-63, 1997.

[10] T-L Chern, C-S Liu, C-F Jong and G-M Yan, "Discrete integral variable structure model following control for induction motor drivers", IEE Proc., Electric Power Applications, Vol. 143, No. 6, pp. 467-474, 1996.

[11] A.S. Murty, S. Parameswaran, K. Ramar, "Design of decentralized variable structure stabilizers for multimachine power systems", Int. J. Elect. Power and Energy Syst., Vol. 18, No. 8, pp. 535-546, 1996

[12] Y. Y. Hsu, and W. C. Chan, "Optimal variable structure controller for the load-frequency control of interconnected hydrothermal power systems", Electrical Power \& Energy Systems, Vol. 6, No 4, October 1984.

[13] X-Y Lu, S. Spurgeon and I. Postlethwaite, "Robust variable structure control of a PVTOL aircraft", Int. J. System Science, Vol. 28, No. 6, pp. 547-558, 1997.

[14] M. Innocenti and A. Thukral, "Robustness of a variable structure control system for maneuverable flight vehicles", $J$. Guidance. Control, and Dynamics, Vol. 20, No. 2, pp. 377-383, 1997

[15] S. Singh, M. Steinberg and R. DiGirolamo, "Variable structure robust flight control system for the F-14", IEEE Trans. Aerospace and Electronic Systems, Vol. 33, No. 1, pp. 77-84, 1997.

[16] M. Zribi, L. Huang and S. Chan, "Variable structure control of two manipulators handling a constrained object", Cybernetics and Systems, Vol. 28, No. 4, pp. 263-286, 1997

[17] K. Lee, S. Coates and V. Coverstone-Carroll, "Variable structure control applied to underactuated robots", Robotica, Vol. 15, No. 3, pp. 313-318, 1997.

[18] Z. Al-Hamouz and H. Al-Duwaish, "A new load frequency variable structure controller using genetic algorithms", Electric Power Systems Research, Vol. 55, pp. 1-6, 2000.

[19] Z. Al-Hamouz and H. Al-Duwaish, "A new variable structure DC motor controller using genetic algorithms", Proc. 1998 IEEE IA Annual Meeting, St. Louis, Missouri, USA, pp. 1669-1673, October 12-16, 1998.

[20] H. Al-Duwaish and Z. Al-Hamouz, "A genetic approach to the selection of the variable structure controller feedback gains", Proc. 1998 IEEE Conference on Control Applications, Trieste- Italy, pp 227-231, Sept. 1-4, 1998.

[21] U. Itkis, Control systems of variable structure, Keter Publishing House, Jerusalem, 1976

[22] V. Utkin and K. Yang, "Methods for constructing discontinuity planes in multidimentional variable structure systems", Autom. and Remote Control", Vol. 39, pp. 1466-1470, 1978.

[23] R. Lippman, "An introduction to computing with neural nets", IEEE ASSP Mag., pp. 4-22, 1987.

[24] D. Rumelhart, G Williams and R Williams, "Learning interna representation by error propagation", Parallel-Distributed Processing, Vol. 1, MIT Press, Cambridge, MA, pp. 318-362, 1986.

[25] Y. N. Yu, Electric power system dynamics, Academic Press, New York, 1983

[26] S. Lee and J. Park, "Design of power system stabilizer using observer/sliding mode, observer/sliding mode-model following and $H_{\infty}$ /sliding mode controllers for small-signal stability study", Electric Power and Energy Systems, Vol. 20, pp. 543-553, 1998. 